ESTUDIO

\title{
PADRES INVOLUCRADOS Y USO DE DROGAS: UN ANÁLISIS EMPÍRICO
}

\author{
Eduardo Valenzuela
}

Este artículo analiza la importancia que tiene el comportamiento de los padres en la prevención del uso de alcohol y drogas entre adolescentes. De todas las variables familiares que predicen conductas de riesgo se destaca especialmente el impacto que tiene el control parental bajo la forma de padres efectivamente involucrados en el comportamiento de sus hijos. También se presenta información complementaria acerca de la estructura familiar y la influencia de los amigos, la que permite precisar el alcance que tiene el estilo parental en la prevención del riesgo adolescente. En su conjunto, el estudio obtiene asociaciones estadísticamente significativas entre involucramiento de los padres y uso de alcohol, marihuana y cocaína, las que mantienen su relevancia incluso cuando se las controla por otros factores determinantes. Los datos de este estudio provienen de las encuestas nacionales de drogas en población escolar realizadas en 2001 y 2003 por el Consejo Nacional Para el Control de Estupefacientes (Conace).

Eduardo Valenzuela. Director del Instituto de Sociología, Pontificia Universidad Católica de Chile.

Estudios Públicos, 101 (verano 2006). 
ـa importancia de la familia en la formación y prevención de conductas de riesgo adolescente ha sido objeto de mucha investigación sociológica de interés. La asociación específica entre influencia parental y uso de alcohol y drogas entre adolescentes ha sido bien documentada para variables como la calidad de la relación padres-hijos (parental attachment), la naturaleza de la supervisión parental (parental monitoring) y los hábitos de consumo de alcohol y drogas que tengan o hayan tenido los padres (Eitle, 2005). La influencia del estilo parental ha sido examinada mediante el concepto de "soporte", que incluye la capacidad de los padres de confiar, animar, aceptar y brindar a los hijos el afecto necesario, y el concepto de “control”, que comprende los esfuerzos por dirigir y orientar el comportamiento de los hijos de una manera aceptable para los padres (Barnes y Farrel, 1992). En el ámbito del control se ha encontrado mucha evidencia consistente acerca del impacto de la supervisión parental en la prevención de conductas de riesgo adolescente: la capacidad de los padres de conocer, atender y supervisar las actividades que los hijos hacen y las relaciones que establecen se identifica actualmente como una de las variables más poderosas en la orientación del comportamiento adolescente. La actitud convencional de control que incluye coerción parental tiene usualmente una relación curvilineal con el comportamiento de riesgo: laxismo y severidad se asocian con mayores problemas conductuales entre los adolescentes. Pero la relación entre supervisión parental y comportamiento adolescente se ha mostrado consistentemente lineal: "Entre todas las dimensiones de control que se miden habitualmente, el mejor y más consistente predictor del comportamiento adolescente es la supervisión parental. Altos niveles de supervisión parental están siempre asociados a menor uso de alcohol, drogas ilícitas, desviación y problemas de conducta en el colegio” (Barnes y Farrel, 1992: 770). Tampoco las formas llamadas inductivas de control que se asocian a la información y consejería parental aparecen como eficaces: "Proveer simplemente a los adolescentes con explicaciones generalizadas de conducta no son efectivas en la prevención del abuso de alcohol y problemas de comportamiento. Se requieren formas más concretas de control parental, tales como proporcionar reglas específicas de conducta y, especialmente, monitorear lo que los adolescentes hacen y con quienes se acompañan fuera de la casa” (Barnes y Farrel, 1992: 770).

El examen de la influencia parental debe distinguirse del problema de la estructura familiar y composición de los hogares. La evidencia acerca de los riesgos en el uso de alcohol y drogas que enfrentan los hijos que no 
viven con ambos padres ha sido recopilada en numerosos estudios (Hoffmann y Johnson, 1998; Thomas, Farrel y Barnes, 1996; McLanahan y Sandefur, 1994). La investigación social ha descubierto que "niños criados en familias no tradicionales, como familias uniparentales y reconstituidas (incluyendo aquellas que agregan hijos de padrastro o madrastra) tienen mayor riesgo de usar drogas” (Eitle, 2004). También se ha aportado evidencia de que "los adolescentes que viven en familias reconstituidas tienen probabilidades muy similares de uso de sustancias que aquellos que viven en familias uniparentales” (Eitle, 2004), aunque la intensidad de estos riesgos cambia según se viva con el padre o con la madre. Los hijos que viven con su padre (sea solo o con otra mujer, que constituyen siempre una fracción muy exigua de la población) presentan mayores riesgos que aquellos que viven con su madre. La conexión entre familias uniparentales o reconstituidas y comportamientos de riesgo adolescente se vincula con tres factores principales: privación económica, movilidad residencial y calidad del soporte y cuidado parental. La literatura sobre el efecto de la estructura familiar encuentra que hasta la mitad del efecto negativo de vivir en una familia uniparental se debe a la penuria económica del hogar que influye sobre los recursos, el tiempo disponible y la interacción familiar (McLanahan y Sandefur, 1994). También la movilidad residencial asociada a la reconstitución familiar se ha observado como un factor crítico en el comportamiento adolescente, bajo una hipótesis que conecta movilidad y desestabilización de los vínculos con la comunidad y los pares (para un recién llegado es más fácil entrar en los grupos "malos" que en los "buenos", que tienden a ser más cerrados y selectivos). La relación entre estructura familiar y calidad de la relación parental es más inestable, aunque se presume que los hijos que no viven con ambos padres tienen relaciones menos comprometidas y menores oportunidades de supervisión parental, así como una predisposición específica a involucrarse más intensamente con el grupo de pares. La relación entre estructura familiar y comportamiento adolescente debe ser calificada, sin embargo, bajo condiciones específicas: el efecto de no vivir con ambos padres puede desvanecerse casi totalmente cuando los niveles de atención y cuidado parental son altos, y de la misma manera, la ventaja de vivir con ambos padres tiende a desaparecer casi totalmente cuando la exposición a amigos que usan alcohol y drogas es muy elevada.

Todos los estudios acerca de comportamientos de riesgo entre adolescentes muestran la importancia del grupo de amigos, y específicamente de la exposición a grupos de pares que usan alcohol y drogas. La existencia 
de amigos cercanos que usan drogas es casi siempre el mejor predictor de prevalencias de uso que se pueda encontrar. El modelo clásico de la influencia de los amigos está tomado de los estudios sobre delincuencia que muestran la importancia de la presión de grupo en la iniciación de la actividad delictiva de los adolescentes. El contacto de adolescentes con baja autoestima y un fuerte deseo de aceptación social con grupos de pares que aprueban y legitiman el comportamiento desviado suele ser el modelo convencional de la influencia de los amigos, que también aparece por doquier en la mentalidad común acerca de las "malas influencias" o los "malos amigos". Mucha de la actividad preventiva en esta materia consiste en dotar a los niños de la capacidad de resistir a la presión de grupo a través del manejo de habilidades emocionales e intelectuales pertinentes. El modelo de la influencia del grupo de pares, sin embargo, se ha mostrado menos eficaz para estudiar el uso de alcohol y drogas, donde los efectos de selección son más importantes: los adolescentes que usan alcohol y drogas tienden a elegir amigos que también lo hacen. No es tanto el contacto con amigos que usan drogas el que induce a iniciarse en su uso, sino que quienes usan drogas tienden a seleccionar amigos que también las usan. "Muchos estudios que comparan los efectos de selección e influencia han encontrado que los primeros son tanto o más poderosos que los segundos en la explicación de las similitudes que existen entre adolescentes y amigos en el uso de cigarrillo y otros problemas de conductas. Otros estudios que controlan por selección, han encontrado alguna evidencia de influencia, pero en una magnitud relativamente baja” (Urberg et al., 2002). Estas conclusiones se obtienen también en Kandel (1978), Fisher y Bauman (1988) y Aseltine (1995): "Mientras los jóvenes parecen estar socializados en comportamientos delictivos por amigos, los factores de selección juegan un papel mucho más importante en las similitudes entre amigos en el caso del uso de drogas”. El balance entre la influencia parental y la que ejercen los amigos suele ser más equilibrado en los estudios de alcohol y drogas que en los estudios sobre comportamiento delictual. Este juego de influencias también fluctúa con la edad, como sugieren las conclusiones de Aseltine (1995): "la influencia parental es crucial en la iniciación temprana en el uso de drogas, mientras que los pares se convierten en una influencia predominante en la iniciación tardía”. Existen estudios, asimismo, que han mostrado que la influencia del grupo de pares depende de variables asociadas al proceso familiar como el nivel de compromiso y supervisión parental, aunque la exposición a ambientes que usan y aprueban generalizadamente el uso de drogas (por ejemplo, colegios o barrios de alta prevalencia donde usualmen- 
te se seleccionan los amigos) puede ejercer una influencia directamente atribuible al grupo de pares. La investigación acerca del uso de drogas oscila en torno a este delicado balance entre familia/amigos en la formación del comportamiento adolescente, y les otorga a ambas variables un peso específico en la determinación del problema.

\section{Importancia de los padres involucrados}

Las encuestas nacionales de drogas en población escolar realizadas por Conace en los años 2001 y 2003 ofrecen datos relevantes acerca de la importancia que tiene la calidad de la relación con los padres en el uso de alcohol y drogas ${ }^{1}$. Conace ha elaborado específicamente una escala de padres involucrados que incluye distintos elementos de atención, cuidado y control parental que se construye a partir de declaraciones que entregan los propios alumnos acerca del comportamiento normal de sus padres. Muchos de los indicadores que componen esta escala se usan corrientemente en la investigación acerca de la asociación entre familia y drogas, especialmente los que se refieren a la supervisión parental de lo que se hace fuera de la casa que constituyen propiamente la variable parental monitoring (los padres siempre saben dónde se encuentran sus hijos, preguntan o esperan que se les diga dónde van cuando salen, están atentos a lo que hacen sus hijos en el colegio y conocen suficientemente a sus amigos). The National Center on Adiction and Substance Abuse, Columbia University, ha mostrado, asimismo, la importancia de ciertos comportamientos parentales dentro de la casa, especialmente la costumbre de comer regularmente juntos, controlar la hora de llegada en las noches y monitorear lo que los hijos ven en la televisión o lo que hacen en internet (CASA: 2005). En su conjunto se utilizará el concepto de "padres involucrados" (hands on parents) para designar la capacidad de los padres para conocer, atender y monitorear el comportamiento de sus hijos de una manera suficiente y continua.

${ }^{1}$ Conace realiza en años impares la Encuesta Nacional de Drogas en Población Escolar sobre la base de una muestra de gran tamaño (alrededor de 58.000 casos). La unidad de muestreo son escolares de octavo básico a cuarto medio, seleccionados mediante un procedimiento probabilístico, bietápico y estratificado por nivel de enseñanza (cursos) y tipo de colegio (municipalizados, subvencionados y pagados), en 87 de las principales comunas del país. El estudio se realiza a través de un cuestionario autoaplicado en sala de clases en un día normal de colegio. Ver Conace, "Quinto Estudio Nacional de Drogas en Población Escolar de Chile, 2003. Informe Final”, 2004. Agradezco especialmente a la Secretaría Ejecutiva de Conace el acceso a sus bases de datos para realizar este estudio. 


\section{ÍNDICE DE PADRES INVOLUCRADOS}

1. Después que sales del colegio o durante los fines de semana, ¿cuántas veces ocurre que tu madre o tu padre no saben dónde estás? Digamos por un período de una hora o más. Siempre saben dónde estoy (1) / A veces no saben / nunca o casi nunca saben dónde estoy (0)

2. En general, ¿alguno de tus padres se fija en los programas que ves en la televisión? Sí (1) / No (0)

3. ¿Cuán atentos están tus padres, o alguno de ellos, respecto de lo que haces en el colegio? (Mucho/Bastante (1) /Poco/Nada (0)

4. Durante los fines de semana, ¿tus padres, o alguno de ellos, te controlan la hora de llegada a tu casa en la noche? Sí (1) / No (0)

5. Cuando sales de la casa en las tardes o en fines de semana, tus padres, o alguno de ellos, te preguntan y/o esperan que tú les digas dónde vas? Sí (0) / No (0)

6. En una semana normal, ¿cuántos días se sientan a comer juntos, tú y tus padres, o alguno de ellos, en la misma mesa? Digamos para almorzar, tomar once comida o comer en la noche. En número de días: 6 o 7 (1) / 5 o menos (0)

Los resultados de este conjunto de indicadores pueden agruparse en un índice donde 0 significa que los padres no tienen ninguno de los comportamientos señalados y 7 que los tienen todos según declaraciones que ofrecen los propios alumnos acerca de sus padres (Alpha de Cronbach de 0,51 en ambos estudios). El promedio de involucramiento parental que declaran alumnos de octavo básico a cuarto medio en el país es de 4,7 en un índice que fluctúa entre 0 y 7 puntos, casi exactamente el mismo promedio en los estudios nacionales de drogas de 2001 y 2003. Alrededor del $60 \%$ de los alumnos obtienen 5, 6 o 7 puntos en el índice, lo que puede considerarse una medida de padres satisfactoriamente involucrados, mientras que cerca del 20\% obtiene 3 o menos puntos, lo que revela inequívocamente padres muy pobremente involucrados en el comportamiento de sus hijos. 
TABLA N ${ }^{\circ}$ 1: $\quad$ ÍNDICE DE PADRES INVOLUCRADOS

\begin{tabular}{lrrrrrrrrr}
\hline & 0 & 1 & 2 & 3 & 4 & 5 & 6 & 7 & \\
\hline 2001 & 0.7 & 2.0 & 5.2 & 11.5 & 19.9 & 25.9 & 24.2 & 10.6 & 100 \\
2003 & 0.8 & 2.1 & 5.5 & 11.4 & 19.6 & 25.7 & 23.4 & 11.6 & 100 \\
\hline
\end{tabular}

Fuente: Conace: estudios nacionales de drogas en población escolar, 20012003.

El comportamiento de este índice arroja resultados esperados en este tipo de variables según se muestra en la Tabla $\mathrm{N}^{\circ}$ 2: las mujeres declaran padres ligeramente más involucrados que los hombres, mientras que el nivel de involucramiento declarado tiende a bajar sistemáticamente con la edad. Los promedios no muestran ningún sesgo socioeconómico relevante, sea que se considere el tipo de establecimiento donde se estudia o el nivel educacional alcanzado por la madre y el padre, lo que contraviene la imagen de desatención parental que se suele imputar en los hogares más pobres. La estructura familiar, por su parte, incide en un sentido ampliamente descrito por la investigación pertinente: aquellos que viven con ambos padres obtienen el promedio de involucramiento parental más alto $(4,9)$, pero el desempeño de familias con madres solas o viviendo con otra pareja distinta del padre es también satisfactorio $(4,6)$, mientras que el promedio cae significativamente cuando se vive con el padre en cualquiera de ambas situaciones $(4,2)$. El nivel de involucramiento declarado, por último, no difiere demasiado según la condición ocupacional de la madre en el hogar: madres que trabajan jornada completa o jornada parcial tienen promedio de 4,6 y 4,7, respectivamente, mientras que madres que no trabajan alcanzan un promedio sólo ligeramente superior $(4,9)$. La diferencia máxima que se puede encontrar oscila entre madres solas que trabajan $(4,5)$ y madres que viven con el padre del alumno y no trabajan $(5,1)$. Debe observarse que la capacidad de los padres de atender y monitorear el comportamiento de los hijos depende también de características de los mismos hijos que es preciso tomar en consideración: antecedentes de hiperactividad y dificultades de disciplina y logro escolar resienten el involucramiento parental en un sentido que no deja de tener cierto dramatismo: los niños que más necesitan de la atención parental terminan por no recibirla. Niños que declaran antecedentes de hiperactividad en estos estudios obtienen un promedio de 4,4 y, al mismo tiempo, los que declaran problemas de disciplina escolar (por ejemplo, hace muchas veces la "cimarra" en un año escolar) alcanzan el promedio más bajo observado $(3,5)$ (datos no mostrados). 
TABLA N ${ }^{\circ}$ 2: $\quad$ PROMEDIOS EN ÍNDICE DE PADRES INVOLUCRADOS Y PREVALENCIAS DE CONSUMO DE MARIHUANA EN ÚLTIMO AÑO SEGÚN CARACTERÍSTICAS SELECCIONADAS

\begin{tabular}{|c|c|c|c|c|}
\hline & $\begin{array}{l}\text { Padres } \\
2001\end{array}$ & $\begin{array}{c}\text { Padres } \\
2003\end{array}$ & $\begin{array}{c}\text { Marihuana } \\
2001\end{array}$ & $\begin{array}{c}\text { Marihuana } \\
2003\end{array}$ \\
\hline Hombre & 4,6 & 4,6 & 16 & 15 \\
\hline Mujer & 4,9 & 4,9 & 13 & 12 \\
\hline Octavo básico & 5,1 & 5,0 & 6 & 5 \\
\hline Primero medio & 4,9 & 4,8 & 11 & 9 \\
\hline Segundo medio & 4,7 & 4,7 & 17 & 15 \\
\hline Tercero medio & 4,5 & 4,6 & 21 & 19 \\
\hline Cuarto medio & 4,4 & 4,4 & 23 & 22 \\
\hline Municipal & 4,8 & 4,8 & 14 & 14 \\
\hline Particular subvencionado & 4,8 & 4,8 & 15 & 13 \\
\hline Particular pagado & 4,7 & 4,7 & 15 & 14 \\
\hline Vive con ambos padres & 4,9 & 4,9 & 13 & 12 \\
\hline Con padre y otra mujer & 4,1 & 4,1 & 22 & 20 \\
\hline Con madre y otro hombre & 4,6 & 4,6 & 19 & 17 \\
\hline Sólo con el padre & 4,2 & 4,2 & 22 & 19 \\
\hline Sólo con la madre & 4,6 & 4,6 & 17 & 16 \\
\hline Con ninguno & 4,1 & 4,0 & 19 & 19 \\
\hline
\end{tabular}

Fuente: Conace: estudios nacionales de drogas en población escolar, 20012003.

Es importante observar que el grado de involucramiento parental está firmemente asociado con la calidad de la relación con la madre y el padre que declaran los alumnos. Mientras más involucrados aparecen los padres, mejor es la relación declarada por sus hijos. Esta relación es enteramente monotónica según se observa en la Tabla $\mathrm{N}^{\circ}$ 3: la proporción que declara tener una excelente relación con su madre y/o su padre aumenta sistemáticamente a medida que se avanza en la puntuación del índice de padres involucrados ${ }^{2}$. Esta relación se mantiene aun cuando se controla por خ curso y sexo (datos no mostrados). La atención, supervisión y control que los padres ejercen sobre sus hijos no aparece asociada a un menoscabo de la relación filial sino que a un mejoramiento ostensible, de modo tal que las dos principales variables del proceso familiar, supervisión (parental monitoring) y cercanía (parental attachment), están íntimamente asociadas.

${ }^{2}$ La pregunta por la relación con los padres incluye las categorías "excelente”, "muy buena", "buena", "no muy buena” y "no se aplica”. Debe notarse que la medida de involucramiento parental no distingue entre padre y madre, mientras que la calidad de la relación parental establece esa distinción. 
TABLA N ${ }^{\circ}$ 3: $\quad$ RELACIÓN CON LA MADRE Y EL PADRE SEGÚN ÍNDICE DE PADRES INVOLUCRADOS

(\% que declara excelente relación con sus padres)

\begin{tabular}{|c|c|c|c|c|c|c|c|c|}
\hline & 0 & 1 & 2 & 3 & 4 & 5 & 6 & 7 \\
\hline \multicolumn{9}{|l|}{ Madre } \\
\hline 2001 & 9 & 16 & 18 & 21 & 29 & 38 & 49 & 62 \\
\hline 2003 & 7 & 14 & 18 & 24 & 31 & 40 & 51 & 66 \\
\hline \multicolumn{9}{|l|}{ Padre } \\
\hline 2001 & 7 & 9 & 12 & 14 & 19 & 25 & 33 & 44 \\
\hline 2003 & 3 & 9 & 11 & 14 & 19 & 24 & 32 & 44 \\
\hline
\end{tabular}

Fuente: Conace: estudios nacionales de drogas en población escolar, 20012003.

La conexión entre padres involucrados y uso de alcohol y drogas puede mostrarse observando las prevalencias de consumo que autodeclaran los alumnos en cada punto del índice de involucramiento parental. La información se presenta para uso de alcohol en último mes y uso de marihuana y cocaína (pasta base o cocaína) en último año. También puede observarse una relación completamente monotónica entre ambas variables: mientras más involucrados aparecen los padres, menores son las declaraciones de consumo. La proporción de quienes han usado alcohol en el último mes oscila en alrededor de $25 \%$ entre quienes tienen padres completamente involucrados y se incrementa a cerca de $60 \%$ entre aquellos que declaran padres pobremente involucrados. Las diferencias en el uso de drogas son todavía más pronunciadas: los alumnos que declaran padres completamente involucrados alcanzan prevalencias de consumo de marihuana de alrededor de $5 \%$ en el último año, mientras que los que reconocen padres pobremente involucrados pueden alcanzar prevalencias que fluctúan en alrededor del 40\%. La amplitud de esta brecha es aún más intensa en el uso de cocaína, que fluctúa entre 1 y $20 \%$ en los extremos de la escala. Es importante notar 乙 también la sensibilidad del índice: cada punto adicional de involucramiento parental va acompañado de una disminución concomitante en la declaración de consumo sea de alcohol, marihuana o cocaína en una relación perfectamente monotónica.

Esta relación entre padres involucrados y uso de alcohol y drogas se mantiene intacta aun cuando se controla por curso (como se muestra en la Tabla $\mathrm{N}^{\circ}$ 5) y sexo: la edad hace disminuir la supervisión parental y aumentar las prevalencias de consumo, pero la asociación entre ambas variables 
persiste en su forma característica tanto en octavo básico como en cuarto medio, aunque es probable que el impacto del involucramiento parental sea mayor en edades más tempranas, como sugieren la amplitud de las brechas que aparecen en octavo básico respecto de las que existen en cursos superiores.

TABLA N ${ }^{\circ}$ 4: $\quad$ PREVALENCIA DE CONSUMO DE ALCOHOL Y DROGAS SEGÚN ÍNDICE DE PADRES INVOLUCRADOS

\begin{tabular}{lrrrrrrrr}
\hline & 0 & 1 & 2 & 3 & 4 & 5 & 6 & 7 \\
\hline $\begin{array}{l}\text { Alcohol } \\
2001\end{array}$ & 64 & 62 & 58 & 52 & 45 & 37 & 31 & 23 \\
2003 & 58 & 59 & 56 & 51 & 45 & 37 & 31 & 25 \\
Marihuana & & & & & & & & \\
2001 & 44 & 40 & 33 & 25 & 18 & 12 & 8 & 4 \\
2003 & 36 & 36 & 27 & 21 & 16 & 11 & 7 & 5 \\
Cocaína & & & & & & & & \\
2001 & 23 & 15 & 12 & 8 & 4 & 3 & 2 & 1 \\
2003 & 20 & 16 & 10 & 7 & 4 & 3 & 2 & 1 \\
\hline
\end{tabular}

Alcohol (tasa de consumo de alcohol en últimos treinta días). Marihuana (tasa de consumo de marihuana en el último año). Cocaína (tasa de consumo de pasta base o cocaína en último año).

Fuente: Conace: estudios nacionales de drogas en población escolar, 20012003.

TABLA N ${ }^{\circ}$ 5: $\quad$ PREVALENCIA DE ALGUNA DROGA (MARIHUANA, COCAÍNA O PASTA BASE) EN ÚLTIMO AÑO SEGÚN ÍNDICE DE PADRES INVOLUCRADOS POR CURSOS

\begin{tabular}{|c|c|c|c|c|c|c|c|c|}
\hline & 0 & 1 & 2 & 3 & 4 & 5 & 6 & 7 \\
\hline \multicolumn{9}{|c|}{ Octavo } \\
\hline 2001 & 39 & 27 & 20 & 16 & 9 & 5 & 3 & 1 \\
\hline 2003 & 35 & 28 & 14 & 11 & 8 & 4 & 3 & 2 \\
\hline \multicolumn{9}{|c|}{ Segundo medio } \\
\hline 2001 & 52 & 48 & 39 & 27 & 20 & 15 & 9 & 8 \\
\hline 2003 & 44 & 36 & 32 & 23 & 19 & 14 & 9 & 5 \\
\hline \multicolumn{9}{|c|}{ Cuarto medio } \\
\hline 2001 & 56 & 45 & 38 & 35 & 27 & 21 & 14 & 8 \\
\hline 2003 & 48 & 55 & 39 & 30 & 25 & 19 & 14 & 12 \\
\hline
\end{tabular}




\section{Parentalidad, estructura familiar y amigos}

¿Cuál es la importancia de la relación parental en el marco de las distintas variables que usualmente están asociadas con el uso de alcohol y drogas? Un análisis multivariado debe incluir como variables de control la edad, el sexo y la educación de los padres. El uso de alcohol y drogas tiende a aumentar progresivamente con la edad en el marco de un proceso en el que el balance de la relación padres-amigos tiende a pasar de un término a otro. La curva característica de uso de marihuana predice un aumento sostenido desde octavo básico hasta cuarto medio (con un punto de aceleración en primero medio que marca el acceso a la enseñanza secundaria) (Tabla $\mathrm{N}^{\circ} 2$ ). Existe evidencia también de que el uso de marihuana continúa aumentando después del ciclo de enseñanza media, con un punto de aceleración característico en los primeros años de enseñanza universitaria, para comenzar a caer después de los 20 años, precisamente cuando la relación familia-amigos pierde su tensión propia del período adolescente (Conace, encuestas nacionales en población general, 2001, 2003, 2002, 2004). Esta relación entre edad y uso de marihuana debe calificarse en este sentido: los desertores escolares tienen tasas de prevalencia hasta tres veces mayores respecto de quienes permanecen en el sistema escolar, pero después del colegio son los universitarios quienes tienen prevalencias más altas que los que se incorporan directamente al trabajo, manteniéndose siempre el desempleo como factor de riesgo en todas las edades (Conace, encuestas 2001 y 2003).

La importancia del sexo radica simplemente en que las tasas de prevalencia son siempre más altas en hombres que en mujeres, cualquiera sea la edad. La curva característica según sexo y edad en el uso de marihuana tiene, sin embargo, su interés particular: las diferencias de género son más bien modestas durante toda la enseñanza media, pero la brecha se expande notoriamente después del colegio. El uso de marihuana continúa aumentando también entre las mujeres después que salen del colegio, pero con un ritmo mucho más pausado que el de los hombres y tiende a caer en una edad ligeramente anterior al punto de inflexión masculino. El sexo, por lo tanto, es una variable que cobra importancia con la edad. Esta expansión de las brechas de género en el comportamiento desviado es un resultado frecuente en la investigación social: muchos investigadores atribuyen esta diferencia al inicio de la edad socialmente reproductiva de la mujer, que inhibe poderosamente su comportamiento de riesgo. Mientras la probabilidad de ser madre es baja, tanto biológica como socialmente considerada, la mujer se compromete casi de igual manera que los hombres en comportamiento de riesgo, pero cuando esa probabilidad aumenta, característicamente después de la enseñanza secundaria, la mujer oblitera y elude esos 
riesgos. La responsabilidad procreativa aparece antes y de manera más viva y perentoria entre las mujeres que entre los hombres. Por último, el nivel socioeconómico es siempre una variable de control necesaria. El uso de marihuana no tiene un sesgo socioeconómico definido, como el que tiene la pasta base, que es más frecuente en los niveles bajos, y el alcohol y la cocaína que prevalece en los niveles de ingreso más altos, al menos cuando se miden tasas de prevalencia, que es la proporción de quienes han usado alguna droga en un período determinado. La intensidad de uso y, sobre todo, la proporción de quienes abusan de marihuana tienen un sesgo socioeconómico mayor, especialmente cuando se toma en consideración la población general, que incluye los desertores escolares. En los estudios de drogas realizado entre escolares, sin embargo, el nivel socioeconómico deja de ser una variable relevante para el caso de las tasas de prevalencia de consumo en marihuana (Conace: 2002, 2004).

Junto con estas variables de control —edad, sexo y educación de los padres (como indicador de nivel socioeconómico) — nuestro modelo de análisis considera las variables que identifican procesos familiares (padres involucrados y calidad de la relación con los padres), estructura familiar (familias con sólo el padre o sólo la madre y familias reconstituidas) y amigos (amigos cercanos que usan marihuana). El involucramiento de los padres se muestra como un índice dicotómico a partir del quinto punto, de manera que se introduce como padres involucrados y padres no involucrados. De la misma manera, la calidad de la relación con ambos padres, que se utiliza como variable próxima de compromiso o cercanía filial, distingue entre quienes tienen excelente relación con ambos padres y los demás. La estructura familiar hace la distinción entre quienes viven con ambos padres y quienes viven sólo con alguno de ellos, y con alguno de ellos y su pareja actual. La variable que involucra a los amigos en este análisis distingue entre quienes declaran tener al menos un amigo cercano que fuma marihuana y quienes no tienen ninguno. La variable dependiente es prevalencia de uso de marihuana en el último año (ha probado o no marihuana en los últimos doce meses).

El modelo 1 (en la Tabla $\mathrm{N}^{\circ}$ 5) considera sólo variables de proceso y estructura familiar controladas por sexo, edad y educación de los padres. Los resultados indican que la variable que mejor predice el uso de marihuana entre adolescentes es el involucramiento de los padres: no vivir con padres involucrados puede aumentar la probabilidad de uso de marihuana en 2,4 veces respecto de quienes viven con padres altamente involucrados. El involucramiento parental aparece como una variable más preeminente que la calidad de la relación con los padres, que marca, no obstante, una asociación también significativa. Los coeficientes de estructura familiar tienen una asociación significativa, pero mucho más débil: no vivir con ambos padres 
ofrece una probabilidad de riesgo, sobre todo cuando se vive con padre o madre y otra pareja (familias reconstituidas). La diferencia entre vivir con el padre o la madre también aparece en el sentido indicado por la investigación social: el riesgo es varias veces mayor cuando se vive con el padre que cuando se vive con la madre, hasta el punto en que vivir sólo con la madre ofrece asociaciones muy débiles con el uso de drogas (datos no mostrados). Debe observarse que el riesgo específico que ofrecen los padres oculta, como todas las asociaciones por lo demás, efectos de autoselección: es común que muchos adolescentes vivan con su padre justamente porque tienen dificultades conductuales y alta propensión al riesgo. Distinciones más sutiles entre el estatuto marital de madre sola también se han realizado, observando efectos diferentes según se trate de madres viudas, separadas o nunca casadas, pero nuestro análisis no contempla estas distinciones. En su conjunto, la asociación entre estructura familiar y uso de drogas se atenúa mucho cuando se controla por involucramiento parental y calidad de la relación con los padres, aunque todavía en estas circunstancias no vivir con ambos padres ofrece alguna probabilidad de riesgo. El modelo 2 considera el efecto de interacción entre padres involucrados y estructura familiar. Los resultados en este caso son menos concluyentes: en condiciones de bajo involucramiento parental, el hecho de vivir sólo con el padre o la madre no añade una probabilidad de riesgo en relación a vivir con ambos padres. Cuando se considera este efecto de interacción con la distinción madre/ padre aparece una asociación significativa: cuando los padres están poco involucrados, vivir sólo con la madre, mas no con el padre, agrega probabilidades de riesgo (datos no mostrados). El hecho de vivir con padre o madre y otra pareja (e incluso de no vivir con ninguno de ellos), sin embargo, no añade mayores probabilidades de riesgo cuando existen dificultades en la relación parental.

Los siguientes modelos, 3 y 4, introducen la variable amigos que usan drogas, lo que aparece como la variable más fuertemente asociada con el uso de marihuana, como ocurre por doquier en todos los análisis similares. Con nuestros datos no es posible examinar la dirección de esta asociación, sea como efecto de autoselección (los que usan drogas tienden a juntarse con amigos que usan drogas) o como efecto de influencia (juntarse con amigos que usan drogas induce a usar drogas). Sólo es posible mostrar que la asociación entre uso de drogas y amigos que la usan es extremadamente poderosa. El último modelo considera el efecto de interacción entre amigos y padres involucrados: el resultado es bastante contraintuitivo porque muestra que en condiciones de bajo involucramiento parental, tener amigos que usan drogas reduce las probabilidades de riesgo. Dorius et al. descubren algo similar: el soporte que entregan los padres no ofrece protección cuando los amigos usan drogas (Dorius, Bahr, Hoffmann y Harmon, 2004). 
TABLA No 6

MODELOS DE REGRESIÓN LOGÍSTICA PARA USO DE MARIHUANA EN ULTIMO AÑO EN POBLACIÓN ESCOLAR

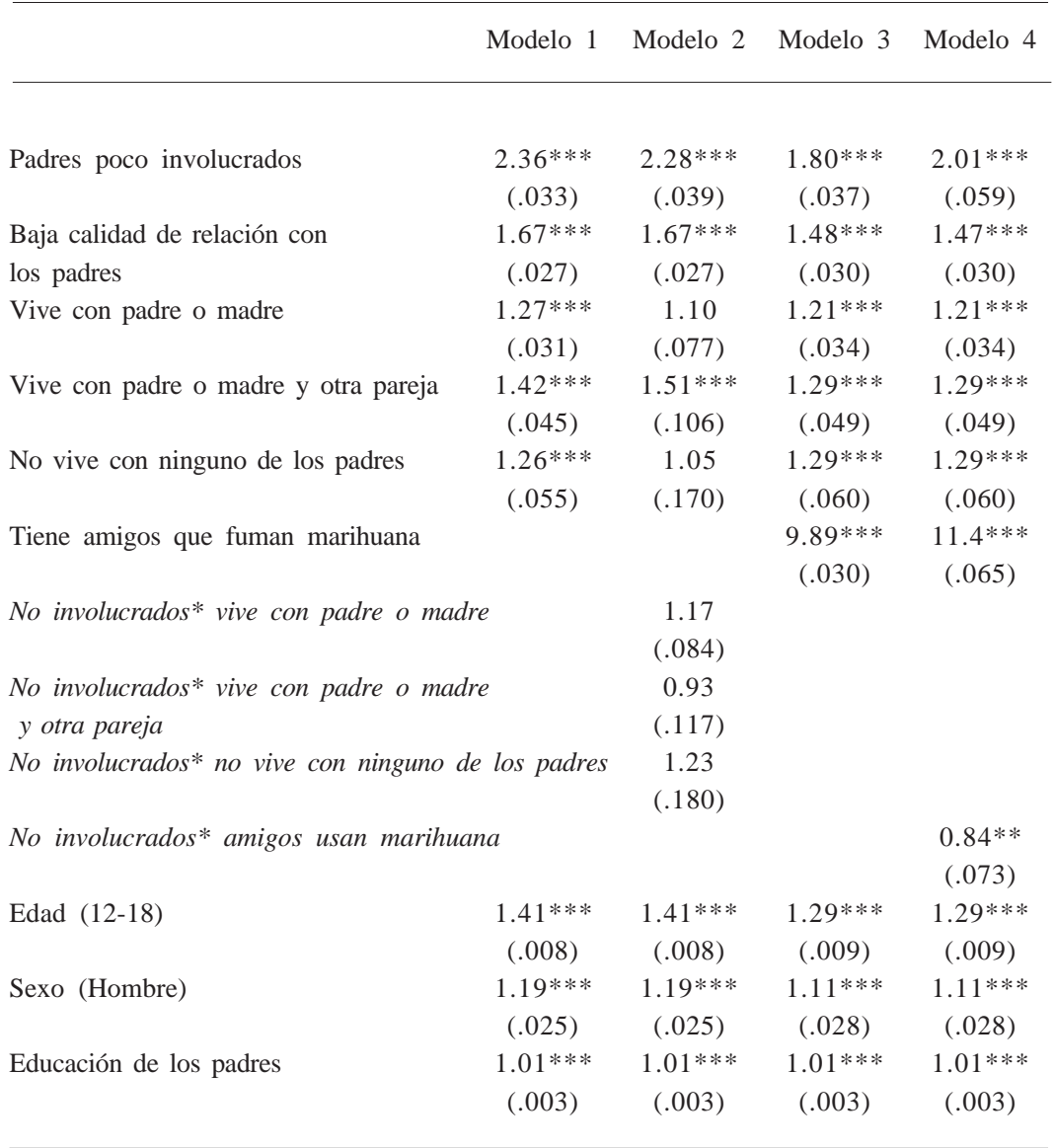

Coeficientes exp (B) y errores estándar entre paréntesis. Niveles de significación: * $\mathrm{p}<0.05 / * * \mathrm{p}<0.01 / * * * \mathrm{p}<0.001$.

Padres involucrados (variable de comparación = padres involucrados que marCan 6 o 7 puntos en índice respectivo). Relación con los padres (variable de comparai. ción = excelente o muy buena relación con ambos padres o con alguno de ellos en caso que faltara el otro). Estructura familiar (variable de comparación = vive con ambos padres). Amigos (variable de comparación = no tiene amigos que usen marihuana). Edad (12-18 años). Educación de los padres (sumatoria de años de estudio del padre y de la madre con duplicación en caso que falte alguno).

O madie con duplicación en caso que falte alguno)

Fuente: Conace: estudios nacionales de drogas en población escolar, 2003. $(\mathrm{N}=58.489)$. 
Los resultados para uso de marihuana no son los mismos cuando se consideran otras sustancias, como alcohol o cocaína. En el caso del alcohol el peso de todas las variables se reduce notablemente, en gran parte porque la medida de uso de alcohol que se utiliza en este caso (ha probado alcohol en los últimos treinta días) no es indicador fuerte de comportamiento de riesgo y la tensión familia/amigos no aparece en su forma característica. La

TABLA N ${ }^{\circ}$ 7: MODELOS DE REGRESIÓN LOGÍSTICA PARA USO DE ALCOHOL, MARIHUANA Y COCAÍNA

\begin{tabular}{lccc}
\hline & $\begin{array}{c}\text { Marihuana } \\
\text { último año }\end{array}$ & $\begin{array}{c}\text { Cocaína } \\
\text { último año }\end{array}$ & $\begin{array}{c}\text { Alcohol } \\
\text { último mes }\end{array}$ \\
\hline Padres poco involucrados & $1.80^{* * *}$ & $1.77^{* * *}$ & $1.49 * * *$ \\
& $(.037)$ & $(.063)$ & $(.021)$ \\
Baja calidad de relación con los padres & $1.48^{* * *}$ & $1.51^{* * *}$ & $1.29^{* * *}$ \\
& $(.030)$ & $(.049)$ & $(.019)$ \\
Vive con padre o madre & $1.21^{* * *}$ & $1.19^{* *}$ & 1.03 \\
& $(.034)$ & $(.055)$ & $(.024)$ \\
Vive con padre o madre y otra pareja & $1.29^{* * *}$ & $1.46^{* * *}$ & 1.06 \\
& $(.049)$ & $(.073)$ & $(.035)$ \\
No vive con ninguno de los padres & $1.29^{* * *}$ & $1.43^{* * *}$ & 0.93 \\
& $(.060)$ & $(.087)$ & $(.045)$ \\
Tiene amigos que usan alcohol / marihuana & $9.89^{* * *}$ & $8.48^{* * *}$ & $4.17 * * *$ \\
& $(.030)$ & $(.054)$ & $(.022)$ \\
Edad (12-18) & $1.29 * * *$ & $1.22^{* * *}$ & $1.27 * * *$ \\
Sexo (Hombre) & $(.009)$ & $(.015)$ & $(.006)$ \\
& $1.11^{* * *}$ & $1.96 * * *$ & $0.95^{* * *}$ \\
Educación de los padres & $(.028)$ & $(.046)$ & $(.019)$ \\
& $1.01^{* * *}$ & $0.93^{* * *}$ & $1.05^{* * *}$ \\
& $(.003)$ & $(.006)$ & $(.002)$ \\
\hline
\end{tabular}

Coeficientes exp (B) y errores estándar entre paréntesis. Niveles de significación: * $\mathrm{p}<0.05 / * * \mathrm{p}<0.01 / * * * \mathrm{p}<0.001$.

Padres involucrados (variable de comparación = padres involucrados que marcan 6 o 7 puntos en índice respectivo). Relación familiar (variable de comparación = excelente o muy buena relación con ambos padres o con alguno de ellos en caso que faltara el otro). Estructura familiar (variable de comparación = vive con ambos padres). Amigos (variable de comparación = no tiene amigos que usan marihuana). Edad (12-18 años). Educación de los padres (sumatoria de años de estudio del padre y de la madre con duplicación en caso que falte alguno).

Fuente: Conace: estudios nacionales de drogas en población escolar, 2003. $(\mathrm{N}=58.489)$.

${ }^{3}$ Sobre los amigos sólo se tiene información acerca de uso de alcohol y marihuana: en los datos sobre cocaína se ha replicado la variable "tiene amigos que usan marihuana”, de manera que se rompe la similitud de sustancias. 
asociación entre padres involucrados, amigos y alcohol, no obstante, se mantiene significativa, mientras que el peso de la estructura familiar se desvanece por completo. También conviene notar que la relación entre padres involucrados/amigos no es tan amplia como en el caso del uso de drogas, lo que significa que el comportamiento de los amigos es algo menos determinante en el uso de alcohol en relación con las variables de procesos familiares. Los factores predictivos en el uso de cocaína (pasta base+cocaína) se asemejan más a los de la marihuana, salvo por el peso notable que adquiere ser hombre en las probabilidades de riesgo: la diferencia de género entre adolescentes, casi inexistente en alcohol y marihuana, se establece nítidamente en pasta base y cocaína. También en este caso las variables de estructura familiar vuelven a ser significativas, con riesgos ligeramente intensificados para quienes no viven con ambos padres.

\section{Conclusión}

Los resultados empíricos confirman el papel central que juegan los padres involucrados en la prevención del uso de alcohol y drogas. Se obtuvo una relación perfectamente lineal entre grado de involucramiento y prevalencias de consumo en todas las sustancias y cualquiera sea la edad de los adolescentes. La fuerza de esta relación se mantiene, en efecto, desde octavo básico hasta cuarto medio prácticamente intacta. En todos los modelos de análisis multivariado, el involucramiento parental arroja coeficientes de asociación que están muy por encima de cualquier otra variable, con la excepción de los amigos. Como se indica largamente en la investigación social, tener padres involucrados, considerada en este análisis como una variante de la supervisión parental, es la variable que prevalece por sobre cualquier otro indicador de procesos familiares, incluso por encima del compromiso o cercanía filial. No basta tener una buena relación con los padres: el desempeño parental en atención, supervisión y control del comportamiento adolescente juega un rol estratégico. Ambas cosas están, por lo demás, perfectamente asociadas como se ha mostrado: padres efectivamente involucrados mejoran la relación con los hijos y ciertas disposiciones específicas del control adolescente no sólo no menoscaban, sino que se asocian directamente con la calidad de la relación parental. Como se ha dicho por doquier la relación padres-hijos no es sólo una relación de conversación, intimidad y confianza, sino también de atención y monitoreo cuyo presupuesto es siempre la desconfianza respecto de lo que los hijos hacen o pueden llegar a hacer. ¿Por qué habría que estar tan atentos a lo que hacen los hijos si no es porque no se confía totalmente en ellos? Este presupuesto raramente se confiesa, pero opera eficaz y sanamente en cual- 
quier relación parental satisfactoria. También esta investigación confirma que la estructura familiar no es una variable decisiva en el comportamiento de riesgo adolescente, y que cuando se controla por las variables de proceso familiar, su impacto se atenúa considerablemente. Vivir con ambos padres ofrece una modesta garantía de conformidad adolescente, siempre significativa estadísticamente, pero en modo alguno definitiva.

La importancia de la atención y supervisión parental está íntimamente conectada con el problema de los amigos, que aparece como la principal fuente de riesgo adolescente. Como se sabe, las teorías de la desviación social reconocen dos modelos clásicos: aquellas que afirman que la desviación es la conducta normal de la adolescencia que sólo puede inhibirse mediante dispositivos de control social, entre los cuales los padres juegan un primerísimo papel (teoría del control), y aquellas que afirman que la desviación es una conducta aprendida en el contacto con ambientes que aprueban e institucionalizan comportamientos de riesgo (teoría de la asociación diferencial). En el primer caso, la familia juega un rol estratégico; en el segundo, son los amigos. La investigación empírica en uso de alcohol y drogas ha logrado detectar la importancia de ambos factores. Los estudios que logran distinguir entre los efectos de selección e influencia de los amigos y pueden ponderar mejor el impacto del grupo de pares, alcanzan un balance en la relación familia/amigos que no permite decidir entre la preponderancia de los factores. Debe observarse que ambas teorías conviven en la mentalidad común: hay padres que otorgan todo el peso de la prueba a su propio desempeño, con independencia del ambiente en que se desenvuelven sus hijos, y otros que observan en el ambiente, y en particular en los amigos, la fuente de todo peligro y riesgo. La investigación es muy indecisa respecto de ambas actitudes. La preocupación por lo que los hijos hacen fuera de la casa y la atención particular que se presta a los ambientes que frecuentan es enteramente razonable: los amigos pueden ofrecer la justificación necesaria para comportamientos de riesgo. La enorme eficacia de la supervisión parental está muy relacionada con la capacidad de los padres de actuar en ese vínculo estratégico. Pero también debe ser cierto que los padres tienen una responsabilidad directa sobre el comportamiento de sus propios hijos con independencia del ambiente que los rodea.

\section{BiBLIOGRAFÍA}

Aseltine Jr., Robert H.: “A Reconsideration of Parental and Peer Influences on Adolescent Deviance”. En Journal of Health and Social Behavior, Volume 36, June 1995.

Barnes, Grace M. y Michael P. Farrel: "Parental Support and Control as Predictors of Adolescent Drinking, Delinquency, and Related Problem Behaviors”. En Journal of Marriage and the Family, Volume 54, November 1992. 
Barnes, Grace M., Michael Farrel y Allen Cairns: "Parental Socialization Factors and Adolescent Drinking Behaviors". En Journal of Marriage and the Family, Volume 48, February 1986.

CASA (The National Center on Addiction and Substance Abuse at Columbia University): "Family Matters: Substance Abuse and the American Family", March 2005.

Conace (Consejo Nacional para el Control de Estupefacientes): “Cuarto Estudio Nacional de Drogas en Población Escolar, 2001”, Documentos de Trabajo, 2002.

Conace (Consejo Nacional para el Control de Estupefacientes): “Quinto Estudio Nacional de Drogas en Población Escolar, 2003”, Documentos de Trabajo, 2004.

Coombs, Robert H. y John Landsverk: "Parenting Styles and Substance Use during Childhood and Adolescence". En Journal of Marriage and the Family, Volume 50, May 1988.

Dorius, Casandra, Stephen Bahr, John Hoffmann, Elizabeth Lovelady Harmon: "Parenting Practices as Moderators of the Relationship Between Peers and Adolescent Marijuana Use”. En Journal of Marriage and the Family, Volume 66, February 2004.

Eitle, David: "The Moderating Effects of Peer Substance Use on the Family StructureAdolescent Substance use Association: Quantity Versus Quality of Parenting”. En Addictive Behaviors, Volume 30, Issue 5, June 2005.

Farrel, Michael P. y Grace M. Barnes: "Family Systems and Social Support: A Test of the Effects of Cohesion and Adaptability on the Functioning of Parents and Adolescents". En Journal of Marriage and the Family, Volume 55, February 1993.

Fisher, Lynn y Karl E. Bauman: "Influence and Selection in the Friend-Adolescent Relationship: Findings from Studies of Adolescent Smoking and Drinking”. En Journal of Applied Social Psychology, Volume 18 (4), 1988.

Hoffmann, John P. y Robert A. Johnson: “A National Portrait of Family Structure and Adolescent Drug Use”. En Journal of Marriage and the Family, Volume 60, August 1998.

Hundleby, John D. y William Mercer: "Family and Friends as Social Environments and Their Relationship to Young Adolescent's Use of Alcohol, Tobacco, and Marijuana”. En Journal of Marriage and the Family, Volume 49, February, 1987.

Kandel, Denisse B.: “Parenting Styles, Drug Use, and Children's Adjustment in Families of Young Adults”. En Journal of Marriage and the Family, Volume 52, February 1990 .

McLanahan, Sara y Gary Sandefur: Growing Up with a Single Parent. Cambridge: Harvard University Press, 1994.

Thomas, George, Michael P. Farrel y Grace M. Barnes: "The Effects of Single-Mother Families and Nonresident Fathers on Delinquency and Substance Abuse in Black and White Adolescents". En Journal of Marriage and the Family, Volume 58, November 1996.

Urberg, Kathryn A., Qing Luo, Coleen Pilgrim, Serdar M. Degirmencioglu: “A Two Stage Model of Peer Influence in Adolescent Substance Use: Individual and Relationship-Specific Differences in Susceptibility to Influence”. En Addictive Behaviors, Volume 28, Issue 7, September 2003. 